\title{
Representation of the International Celestial Reference System (ICRS) by different sets of selected radio sources
}

\author{
E. F. Arias ${ }^{1, \star}$ and S. Bouquillon ${ }^{2}$ \\ 1 Bureau International des Poids et Mesures, Pavillon de Breteuil, 92312 Sèvres Cedex, France \\ e-mail: farias@bipm.org \\ 2 Observatoire de Paris - SYRTE, 66 Av. de l'Observatoire, 75014 Paris, France \\ e-mail: sebastien. bouquillon@obspm.fr
}

Received 24 July 2003 / Accepted 17 November 2003

\begin{abstract}
To contribute to the maintenance and extension of the ICRF, we have studied representations of the ICRS by different sets of selected radio sources. The axes of the ICRS are realized by the coordinates of 212 sources in the ICRF qualified as the "defining" objects of the frame. They have been selected by the WGRF of the IAU by applying quality criteria to VLBI observations from 1975-1995. A different selection of sources has been proposed by Feissel-Vernier based on an analysis of time series of radio source coordinates over the period 1989-2002. We compare the quality of the realization of the ICRS by the two sets of selected sources.
\end{abstract}

Key words. reference systems - techniques: interferometric

\section{Introduction}

The International Astronomical Union (IAU) recommended (1994) the adoption of a celestial reference system realized on the basis of precise coordinates of extragalactic radio sources observed with the technique of Very Long Baseline Interferometry (VLBI). The celestial reference system of the International Earth Rotation Service (IERS) (Arias et al. 1995) has been adopted as the International Celestial Reference System (ICRS); the ICRS is determined in the radio frequencies by the coordinates of the radio sources in the International Celestial Reference Frame (ICRF); the Hipparcos catalogue is the ICRS realization in the optical frequencies (Kovalevsky et al. 1997).

The first realization of the ICRF (Ma et al. 1998) is the result of the effort of the Working Group on Reference Frames (WGRF) of the IAU. The extension of the ICRF, published under the name ICRF-Ext.1 (IERS 1999) made the frame more dense by including of some tens of new radio sources. Three sets of criteria were adopted by the WGRF to classify the sources in the ICRF: quality of data and observational history; consistency of coordinates derived from subsets of data; repercussions of source structure. The so-called "defining sources" were used to align the axes of the resulting catalogue to the ICRS.

Analyses developed by Feissel-Vernier (2003) show that it is possible to make a judicious classification of radio sources

\footnotetext{
* Associate Astronomer to the Observatoire de Paris - SYRTE.
}

based on statistical studies of their coordinate time series, among other tests. Comparing the sets issued from the two classifications, it has been found that the criteria often diverge, and consequently the sources in each set are different.

We present an analysis of VLBI celestial reference frames by using the sources selected by the WGRF to establish the ICRS on one side, and those from the Feissel-Vernier selection $(\mathrm{F}-\mathrm{V})$ on the other. We compare the results obtained from both sets and conclude the superiority of F-V criteria of classification of radio sources for a better representation of the ICRS.

\section{Data}

Independent VLBI analysis groups regularly submit positions of the IERS catalogues of radio source for validation. The process of validation is performed at the ICRS Product Centre at the Paris Observatory, and it includes the analysis of the formal uncertainties of the radio source coordinates and the comparison of the individual extragalactic reference frames to the ICRF. The latest results were published in the IERS Annual Report 2002 (IERS 2003). From the latest submissions we selected two catalogues determined from independent VLBI analysis: RSC (BKGI) 02 R 01, and RSC (IAA) 02 R 03.

The celestial frame RSC (BKGI) $02 \mathrm{R} 01$ is the VLBI global solution computed at the BKG VLBI Analysis Centre in cooperation with the VLBI group at the University of Bonn. A world-wide set of VLBI observations over the 
Table 1. Analyzed VLBI celestial reference frames: $n$ is the number of sources, $m$ is the median of the position formal uncertainty, in $0.001^{\prime \prime}$.

\begin{tabular}{lccccccccccccc}
\hline \hline \multirow{2}{*}{ Frame } & \multirow{2}{*}{ Total } & \multicolumn{2}{c}{ Defining } & \multicolumn{2}{c}{ Candidate } & \multicolumn{2}{c}{ Other } & \multicolumn{3}{c}{ New } & \multicolumn{2}{c}{ Addit. } & \multicolumn{2}{c}{ Dec } \\
& $n$ & $n$ & $m$ & $n$ & $m$ & $n$ & $m$ & $n$ & $m$ & $n$ & $m$ & $\left(^{\circ}\right)$ \\
\hline RSC (BKGI) 02 R 01 & 594 & 209 & 0.13 & 216 & 0.13 & 102 & 0.04 & 44 & 0.24 & 23 & 0.76 & $-81 ;+84$ \\
RSC (IAA) 02 R 03 & 323 & 85 & 0.11 & 115 & 0.10 & 68 & 0.06 & 47 & 0.23 & 8 & 0.45 & $-44 ;+84$ \\
\hline
\end{tabular}

period 1984-2001 has been processed with the software CALC9.12/SOLVE release 2001.12.21.

The celestial frame RSC (IAA) 02 R 03 has been established by the VLBI group of the IAA Laboratory of New Methods in Astrometry and Geodynamics (NMAG) in St. Petersbourg. The solution was obtained with the software QUASAR (version QUASAR_LSC). NEOS data for the period 1993-2001 was analyzed for this solution. In both cases constraints have been applied to align the axes to the ICRS.

The characteristics of the two frames are shown in Table 1. The number of sources in each ICRF category is given, with the median of the position formal uncertainties. "Defining", "candidate" and "other" follow the traditional ICRF classification of sources (Ma et al. 1998); "new" stands for the sources in ICRF-Ext.1 which were not in the first version of the frame; "additional" stands for those sources observed in recent VLBI programmes, not listed in ICRF-Ext.1.

\section{Algorithm}

It has been shown that the radio source coordinate differences $(\Delta \alpha, \Delta \delta)$ between two frames can be modelled by an algorithm that takes into account the global rotation between the axes of the frames and the deformations; these later can be represented by three parameters: two slopes, one in right ascension as a function of the declination, another in declination as a function of the declination, and a bias in declination representing a relative shift of the principal planes of the frames (IERS 1996). This algorithm of comparison is the one currently used at the ICRS Product Centre; the angles $A_{1}, A_{2}, A_{3}$, represent the small angles around the axes $x, y, z$, respectively; $D_{\alpha}$ is the slope in right ascension as a function of the declination, $D_{\delta}$ is the slope in declination as a function of the declination, $B_{\delta}$ is the bias between the principal planes of the frames compared.

$A_{1} \tan \delta \cos \alpha+A_{2} \tan \delta \sin \alpha-A_{3}+D_{\alpha}\left(\delta-\delta_{0}\right)=\Delta \alpha$

$-A_{1} \sin \alpha+A_{2} \cos \alpha+D_{\delta}\left(\delta-\delta_{0}\right)+B_{\delta}=\Delta \delta$.

The parameters are evaluated by a weighted least squares fit; the equations are weighted by using the inverse of the variance of the coordinate differences. To render the weights realistic, formal uncertainties of coordinates in the frames smaller than 0.01 mas are set to this value for the assignment of weights.

\section{Catalogue comparison}

We have used ICRF-Ext.1 as a reference for the comparisons. In all cases the fitted parameters are those to transform coordinates in the individual frames into ICRS.
Two comparisons have been performed by using different sets of sources:

(a) ICRF classification of sources;

(b) Feissel-Vernier (F-V) classification of sources.

Whilst in the ICRF work sources have been classified by applying criteria based on the quality of the data, the observational history of the radio sources, the consistency of coordinates derived from different data subsets and the evidence of radio structure, F-V classification is mainly supported by the observational history of sources and the analysis of time series of radio source coordinates.

The ICRF has three types of sources, in decreasing order of astrometric quality: defining, candidate and other. The classification of sources in the ICRF has been done over a total of the 608 sources in the first realization of the frame; 212 fulfilled the criteria for the defining sources, 294 were classified as candidate sources and 102 had excessive position variation, and were included in the category "other". The alignement of the axis of the various realizations of the system is assessed by the defining sources. The first extension of ICRF, ICRF-Ext.1 (IERS 1999) densified the frame by adding coordinates of 59 "new" sources; the coordinates and uncertainties of the defining sources remained the same as in ICRF; for candidate and other sources the positions and uncertainties reflect the changes in the data set and the analysis.

The F-V analysis is based on time series of sources coordinates produced by an independent analysis center (USNO). It consists of a two step process on some 700 radio sources observed in the period 1989.5-2002.4. A first selection, made on the basis of continuity criteria for one-year weighted average coordinates, retained 362 sources. From the ICRF side, $67 \%$ of defining sources, $44 \%$ of candidate and $85 \%$ of others are in the F-V selection. The second step of the procedure consists of selecting a set of stable sources by applying different criteria and looking at their intersection. The originality with respect to previous classifications is that F-V studies the stability of radio-source positions by statistical analyses of the time series of the yearly averaged coordinates (standard deviation, Allan standard deviation, normalized linear drift). A subset of 199 sources are qualified as "stable" after these tests.

The set of F-V selected sources has the following components from the ICRF: 81 are defining sources, 68 are candidate sources, 49 are in the list of other sources. This means that $75 \%$ of the sources selected by F-V are either defining in the ICRF or candidates, which are potentially defining in the future. 
$90^{\circ}$

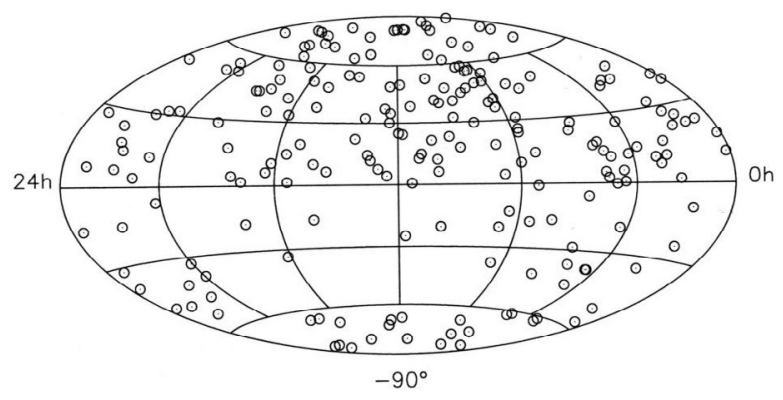

$90^{\circ}$

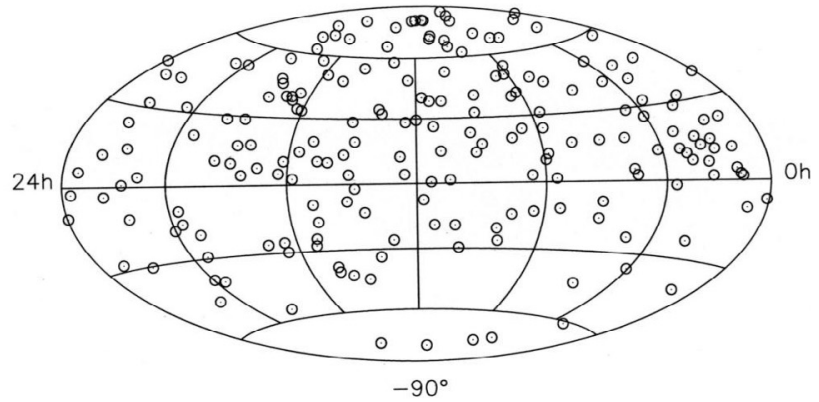

Fig. 1. distribution of the ICRF defining sources (left) and of the F-V stable sources (right).

Table 2. Total $(N)$, ICRF defining (Def.) and F-V stable (Stab.) sources in the individual frames analyzed. The same information is given also for ICRF-Ext.1.

\begin{tabular}{llrr}
\hline \hline Frame & $N$ & Def. & Stab. \\
\hline RSC (BKGI) 02 R 01 & 594 & 209 & 198 \\
RSC (IAA) 02 R 03 & 323 & 85 & 138 \\
ICRF-Ext.1 & 667 & 212 & 81 \\
\hline
\end{tabular}

The number of defining ICRF sources and of stable F-V sources are shown in Table 2.

(a) Catalogue comparison using ICRF defining sources

Both individual frames determined at the BKG and at the IAA have been compared to ICRF-Ext.1 using Eqs. (1). The comparison was done on the basis of the ICRF defining sources present in each frame. The number of defining sources in the comparison is shown in the column "Def." of Table 2. The global relative orientation and the deformation parameters between ICRF and the individual frames were estimated by a weighted least squares fit; they are given in Tables 3 and 4.

Following the usual practice in VLBI work, constraints have been applied to align the axes of the individual catalogues to those of ICRS; the angles in Table 3 show that they are aligned better than 60 microarcsec. The deformation parameters in Table 4 indicate that no deformations are detected in the individual frames relative to the sphere defined by the ICRF.

\section{(b) Catalogue comparison using F-V stable sources}

The previous analysis was applied to catalogue comparison using the F-V selected sources present in each individual reference frame analyzed. The number of stable sources in the comparison is shown in the column "Stab." of Table 2. Tables 5 and 6 show the result of these comparisons.

\section{Discussion}

We have used two VLBI individual frames in our analysis. The radio source coordinates in each catalogue have been calculated using different VLBI analysis software. The sky cover-

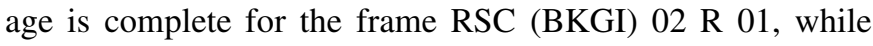

RSC (IAA) 02 R 03 is rather incomplete in the southern celestial hemisphere. Concerning the quality of the positions, as indicated by the median of the position formal uncertainties in Table 1, we can conclude that both frames are equivalent. The number of ICRS defining sources and of F-V stable sources listed in the two frames is shown in Table 2; an increase of sources used in the comparison is clear for the IAA catalogue when the F-V set is used (62\% more objects than the ICRF common defining sources). Figure 1 plots the distribution of the ICRF defining sources and of the F-V stable sources.

Results of catalogue comparison to ICRF in Tables 3 and 5 show that the set of stable sources selected by Feissel-Vernier (2003) defines the axes of the ICRS better than the defining sources in the ICRF; in both individual frames the directions of the axes are closer to those of ICRS when they are represented by the stable F-V sources. The same improvement is observed in the uncertainties of the fitted rotation angles $A_{1}, A_{2}, A_{3}$.

Tables 4 and 6 show the deformations of the individual frames relative to ICRF, represented by the slopes in right ascension and declination as a function of the declination, and by the bias of the reference plane of the catalogues with respect to that of the ICRF. The values of the slopes $D_{\alpha}$ and $D_{\delta}$ indicate for both sets of sources that the radio source coordinates in the two catalogues do not present any systematic effect varying with the declination. No significant bias of the principal plane is observed for RSC (IAA) 02 R 03 by any of the sets of sources. In the case of RSC (BKGI) $02 \mathrm{R} 01$, a bias of -0.016 mas is seen when the F-V selected sources are used for the comparison. This effect is due mainly to the fact that the 199 F-V stable sources are rather unevenly distributed south of the celestial equator, which is not the case for ICRF and for RSC (BKGI) 02 R 01.

The weighted post-fit mean residuals in $\alpha \cos \delta$ and in $\delta$ (represented by $r_{\alpha}, r_{\delta}$ ) for both comparisons (a) and (b) are given in Table 7. The values indicate that the quality of the fit is better by a factor of two for the stable sources selected by F-V.

\section{Conclusion}

The ICRS is determined by VLBI radio source positions in the international reference frame, ICRF. The IAU WGRF made a classification of sources in the ICRF by adopting the criteria in Ma et al. (1998). In the ICRF (as well as 
Table 3. Comparison of individual VLBI frames to ICRF by using common ICRF defining sources. $A_{1}, A_{2}, A_{3}$ are the rotation angles which transform coordinates from the individual frames into ICRF; unit is $0.001^{\prime \prime}$.

\begin{tabular}{llll}
\hline \hline Frame & $A_{1}$ & $A_{2}$ & $A_{3}$ \\
\hline RSC (BKGI) 02 R 01 & $+0.036 \pm 0.016$ & $+0.004 \pm 0.016$ & $-0.053 \pm 0.021$ \\
RSC (IAA) 02 R 03 & $-0.009 \pm 0.024$ & $+0.018 \pm 0.025$ & $-0.023 \pm 0.036$ \\
\hline
\end{tabular}

Table 4. Comparison of individual VLBI frames to ICRF by using common ICRF defining sources. $D_{\alpha}, D_{\delta}$ are the slopes in right ascension and declination respectively, $B_{\delta}$ is the bias in declination; units are $0.001^{\prime \prime} / \mathrm{deg}$ for the slopes, $0.001^{\prime \prime}$ for the bias.

\begin{tabular}{llll}
\hline \hline Frame & $D_{\alpha}$ & $D_{\delta}$ & $B_{\delta}$ \\
\hline RSC (BKGI) 02 R 01 & $-0.001 \pm 0.001$ & $+0.000 \pm 0.000$ & $+0.002 \pm 0.017$ \\
RSC (IAA) 02 R 03 & $+0.001 \pm 0.001$ & $+0.001 \pm 0.001$ & $-0.005 \pm 0.038$ \\
\hline
\end{tabular}

Table 5. Comparison of individual VLBI frames to ICRF by using common F-V stable sources. $A_{1}, A_{2}, A_{3}$ are the rotation angles which transform coordinates from the individual frames into ICRF; unit is $0.001^{\prime \prime}$.

\begin{tabular}{llll}
\hline \hline Frame & $A_{1}$ & $A_{2}$ & $A_{3}$ \\
\hline RSC (BKGI) 02 R 01 & $+0.029 \pm 0.010$ & $-0.002 \pm 0.009$ & $-0.017 \pm 0.011$ \\
RSC (IAA) 02 R 03 & $-0.003 \pm 0.012$ & $+0.012 \pm 0.011$ & $-0.007 \pm 0.014$ \\
\hline
\end{tabular}

Table 6. Comparison of individual VLBI frames to ICRF by using common F-V stable sources. $D_{\alpha}, D_{\delta}$ are the slopes in right ascension and declination respectively, $B_{\delta}$ is the bias in declination; units are $0.001^{\prime \prime} / \mathrm{deg}$ for the slopes, $0.001^{\prime \prime}$ for the bias.

\begin{tabular}{llll}
\hline \hline Frame & $D_{\alpha}$ & $D_{\delta}$ & $B_{\delta}$ \\
\hline RSC (BKGI) 02 R 01 & $-0.001 \pm 0.000$ & $+0.000 \pm 0.000$ & $-0.016 \pm 0.011$ \\
RSC (IAA) 02 R 03 & $+0.000 \pm 0.000$ & $+0.000 \pm 0.000$ & $+0.000 \pm 0.015$ \\
\hline
\end{tabular}

Table 7. Weighted mean residuals $r_{\alpha}$ and $r_{\delta}$ after fitting the global rotations and the deformation parameters between individual frames and ICRF by using the common ICRF defining sources (Def.), and by using the common F-V stable sources (Stab.); unit is 0.001".

\begin{tabular}{lcccc}
\hline \hline \multirow{2}{*}{ Frame } & \multicolumn{2}{c}{$r_{\alpha}$} & \multicolumn{2}{c}{$r_{\delta}$} \\
& Def. & Stab. & Def. & Stab. \\
\hline RSC (BKGI) 02 R 01 & 0.22 & 0.13 & 0.28 & 0.13 \\
RSC (IAA) 02 R 03 & 0.21 & 0.13 & 0.31 & 0.15 \\
\hline
\end{tabular}

in ICRF-Ext.1), the reference system is represented by the 212 defining sources. Analyses recently published show that a set of 199 stable sources can be selected by applying other criteria (Feissel-Vernier 2003); 81 of them are defining objects in the ICRF. The originality of the F-V selection is the analysis of the stability of the time series of the yearly averaged radio source coordinates.

We have compared two individual reference frames obtained from independent VLBI analysis and having a different space distribution of objects to ICRF using the ICRF defining sources on one side, and the F-V stable sources on the other side. The results show that in either case, the orientation of the axes of the ICRS is better realized by using the set of stable sources selected at Feissel-Vernier (2003). This shows that in the selection of the more stable sources for the realization of the celestial reference system, statistical tests on the time-varying behaviour of source coordinates should be included. VLBI observations from 1979-1995 were used in the construction of the ICRF and in the classification of its radio sources. The selection of sources by Feissel-Vernier is applied to observations during the period 1989.5-2002.4, indicating that limiting the time span of observations to the last ten years favours the quality of the frame. It is desirable to densify the set of stable F-V sources south of $-50^{\circ}$ declination.

\section{References}

Arias, E. F., Charlot, P., Feissel, M., \& Lestrade, J.-F. 1995, A\&A, 303, 604

Kovalevsky, J., Lindegren, L., Perryman, M. A. C., et al. 1997, A\&A, 323,620

Ma, C., Arias, E. F., Eubanks, T. M., et al. 1998, AJ, 116, 516

IERS 1996, International Earth Rotation Service Annual Report 1995 (Observatoire de Paris, Paris), II-19

IERS 1999, International Earth Rotation Service Annual Report 1998 (Observatoire de Paris, Paris), 87

IERS 2003, International Earth Rotation and Reference Systems Service Annual Report 2002 (BKG, Frankfurt am Main), 56

Feissel-Vernier, M. 2003, A\&A, 403, 105 